\title{
Performance of Generalized Selection Combining for Mobile Radio Communications With Mixed Cochannel Interferers
}

\author{
Chi Ming Lo and Wong Hing Lam, Senior Member, IEEE
}

\begin{abstract}
The performance of generalized selection combining (GSC) space diversity for mobile radio systems in the presence of multiple cochannel interferers is studied. Two cochannel interference models are considered: 1) $L$ cochannel interferers consisting of $L-N$ Nakagami- $m$ interferers and $N$ Rayleigh interferers and 2) $L$ cochannel interferers in which each interferer follows Nakagami- $m$ distribution for a fraction of time and Rayleigh distribution for the remaining of time. The fading parameters of the Nakagami- $m$ interferers are limited to integer values only. The desired signal is assumed to be Rayleigh faded. Also, all the desired signals and the cochannel interferers received on each branch are independent of each other. Closed-form expressions are derived for the probability density functions (pdfs) of the instantaneous signal-tointerference power ratio (SIR) at the output of the GSC for the two cochannel interference models. Using these SIR pdfs, closed-form expression for evaluating the outage probability and the average bit error probability (BEP) are subsequently derived. A differential phase-shift keying scheme is considered in the derivation. Numerical results showing the influences of various system parameters on the outage probability and the average BEP are then presented.
\end{abstract}

Index Terms-Cochannel interference, generalized selection combining (GSC), Nakagami- $m$ fading, Rayleigh fading.

\section{INTRODUCTION}

$\mathbf{I}^{\mathrm{N}}$ $\mathrm{N}$ MOBILE radio communications, the presence of multipath fading deteriorates system performance and cochannel interference limits system capacity. Space diversity combining, which combines multiple replicas of received signals, has long been recognized as an effective compensation technique for combating multipath fading and cochannel interference [1], [2]. Two methods to combine these multipath components are maximal ratio combining (MRC) and selection combining (SC). MRC is known as the optimal combining technique at the expense of implementation complexity. SC is considered as the simplest method, but it achieves much lower diversity gain than MRC. Recently, Kong et al. (see, e.g., [3] and [4]) published a number of papers bridging the gap between these two extremes (MRC and SC) by introducing generalized selection combining (GSC), which optimally combines the $D_{c}$ largest signal(s) out of $D$ available diversity branch signals.

Previous works have studied the outage probability and the average BEP of GSC diversity systems over various fading

Manuscript received May 13, 2001; revised August 17, 2001.

The authors are with the Department of Electrical and Electronic Engineering,

The University of Hong Kong, Hong Kong, China (e-mail: cmlo@ieee.org).

Publisher Item Identifier S 0018-9545(02)00447-4. channels. Eng et al. [4] derived a closed-form expression for the average BEP of coherent and differential binary phase-shift keying (BPSK/DPSK) for GSC over Rayleigh fading channels for $D_{c}=2$ and 3 and arbitrary $D$. Alouini and Simon [5] studied the outage and the average error probabilities of $M$-ary PSK (MPSK) and $M$-ary quadrature amplitude modulation (MQAM) for GSC over Rayleigh fading channels. In [6], they presented an average BEP analysis of coherent binary modulations for GSC over Nakagami- $m$ fading channels for $D_{c}=2$ and $D=3$ and 4 . In [7], they then extended the average BEP analysis to include MPSK and MQAM for arbitrary $D_{c}$ and $D$. Ma and Chai [8] presented an error probability analysis for GSC over Nakagami- $m$ fading channels for various coherent and noncoherent modulation schemes and nonindependent identically distributed (i.i.d.) branch fading statistics. To the best of the authors' knowledge, no performance analysis of GSC diversity systems over fading channels with cochannel interference has been reported in literature.

Cochannel interferers are usually assumed to follow a single fading distribution in the literature. However, since cochannel interferers are traveling in very different paths, they are most probably experiencing different kinds of fading distributions. In addition, a single interferer may follow different fading distributions at different points in time due to the rapidly changing nature of mobile radio environment. It is therefore of interest to investigate the performance of GSC diversity systems under these two situations.

In this paper, we thus derive closed-form expressions to evaluate the performance of GSC diversity systems over fading channels with multiple cochannel interferers. Two cochannel interference models are considered: 1) $L$ cochannel interferers consisting of $L-N$ Nakagami- $m$ interferers and $N$ Rayleigh interferers and 2) $L$ cochannel interferers in which each interferer follows Nakagami- $m$ distribution for a fraction of time and Rayleigh distribution for the remaining of time. The desired signal is assumed to be Rayleigh faded. With the assumption of an interference-limited environment, the probability density functions (pdfs) of the instantaneous signal-to-interference power ratio (SIR) at the GSC output are then derived for both cochannel interference models. Using these new SIR pdfs, the outage probability and the average BEP are subsequently derived. Note that DPSK scheme is assumed.

The outline of this paper is as follows. The system model is described in Section II. In Section III, we will briefly describe the two cochannel interference models. The performance of GSC diversity systems over fading channels is then derived in 


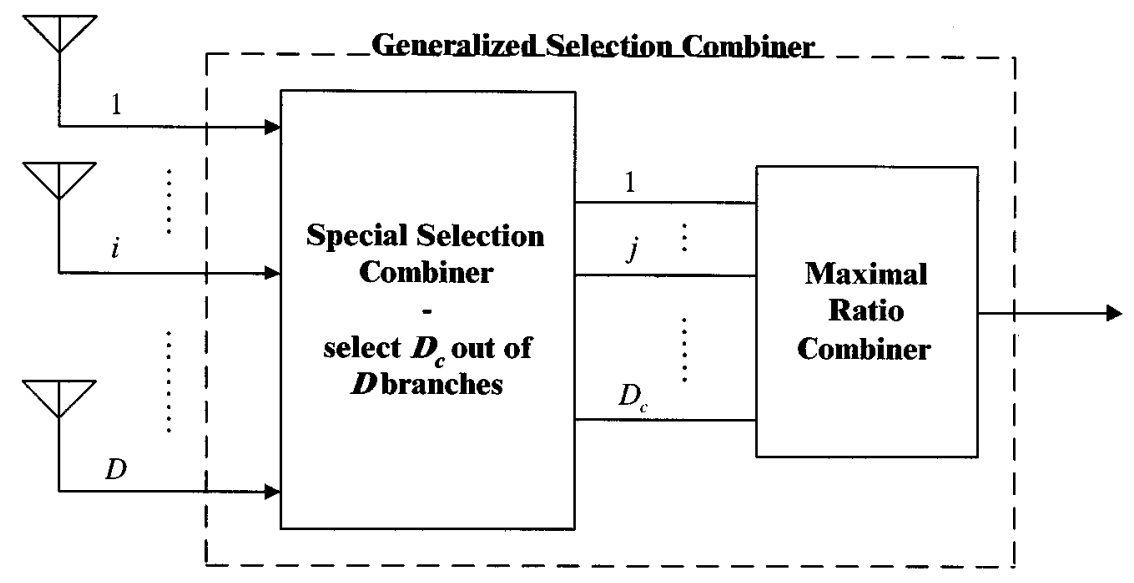

Fig. 1. Block diagram of a generalized selection combiner.

Section IV for the two cochannel interference models. Numerical results are shown in Section V and conclusions are summarized in Section VI.

\section{SySTEM MODEL}

In a cellular radio environment, there is usually a number of cochannel interferers from different cells at the receiver. Typically, the same cochannel interferers are present on each diversity branch [14], [15]. In this paper, a GSC diversity combiner is considered and its block diagram is shown in Fig. 1. As can be seen in Fig. 1, a GSC combiner consists of a special SC (SSC) combiner and a conventional MRC combiner. Considering the MRC portion in Fig. 1, we know from [6] that the instantaneous SIR at the MRC output (or the GSC output) $\gamma$ can be shown to be given by

$$
\gamma=\sum_{j=1}^{D_{c}} \gamma_{j}
$$

where $\gamma_{j}$ is the instantaneous SIR at the $j$ th SSC output branch. Note that we assume $\gamma_{1}>\gamma_{2}>\cdots>\gamma_{D_{c}}$. Therefore, $\gamma$ can also be written as

$$
\gamma=\sum_{j=1}^{D_{c}} \max _{j}(\mathbf{X})=\max _{1}(\mathbf{X})+\max _{2}(\mathbf{X})+\cdots+\max _{D_{c}}(\mathbf{X})
$$

where $\max _{p}(\mathbf{W})$ is the $p$ th largest element in the vector $\mathbf{W}$ and

$$
\begin{aligned}
\mathbf{X} & =\left[\begin{array}{llllll}
x_{1} & x_{2} & \cdots & x_{i} & \cdots & x_{D}
\end{array}\right] \\
& =\left[\begin{array}{llllll}
S_{1} / Y & S_{2} / Y & \cdots & S_{i} / Y & \cdots & S_{D} / Y
\end{array}\right] \\
& =\mathbf{S} / Y
\end{aligned}
$$

Note that $x_{i}$ and $S_{i}$ are, respectively, the instantaneous SIR and the instantaneous desired signal power on the $i$ th diversity branch at the SSC combiner input. Since the same cochannel interferers are present on each diversity branch, we assume that $Y$ is the instantaneous power of the resultant cochannel interferer per diversity branch. Hence, the instantaneous SIR at the GSC output $\gamma$ can be written as

$$
\begin{aligned}
\gamma & =\max _{1}\left(\frac{\mathbf{S}}{Y}\right)+\max _{2}\left(\frac{\mathbf{S}}{Y}\right)+\cdots+\max _{D_{c}}\left(\frac{\mathbf{S}}{Y}\right) \\
& =\frac{\max _{1}(\mathbf{S})}{Y}+\frac{\max _{2}(\mathbf{S})}{Y}+\cdots+\frac{\max _{D_{c}}(\mathbf{S})}{Y} \\
& =\frac{\sum_{k=1}^{D_{c}} \max _{k}(\mathbf{S})}{Y}=\frac{S}{Y}
\end{aligned}
$$

where $S$ is the instantaneous desired signal power at the GSC output.

\section{COCHANNEL INTERFERENCE MODELS}

In consideration of a mobile radio system, where each cochannel interferer is either modeled by Nakagami- $m$ or Rayleigh distribution, the pdf of the instantaneous interference power $Y_{i}$ of the $i$ th interferer is given by [2], [9]

$$
\begin{aligned}
& p_{\text {Nak }}\left(y_{i}\right)=\left(\frac{m_{i}}{\beta_{i}}\right)^{m_{i}} \frac{y_{i}^{m_{i}-1}}{\Gamma\left(m_{i}\right)} \exp \left(-\frac{m_{i}}{\beta_{i}} y_{i}\right) \\
& \text { for Nakagami- } m \text { cochannel interferer }
\end{aligned}
$$

or

$$
\begin{aligned}
p_{\text {Ray }}\left(y_{i}\right)= & \frac{1}{\beta_{i}} \exp \left(-\frac{y_{i}}{\beta_{i}}\right) \\
& \text { for Rayleigh cochannel interferer }
\end{aligned}
$$

where $m_{i}$ is the $i$ th interferer's fading severity parameter, $\beta_{i}$ is the average $i$ th interferer's power, and $\Gamma(\cdot)$ denotes the gamma function [10]. The corresponding characteristic functions (CFs) for the Nakagami- $m$ and Rayleigh cochannel interferers are, respectively, given by [11]

$$
\begin{aligned}
& \Phi_{\mathrm{Nak}}(z)=\left(\frac{m_{i} / \beta_{i}}{z+m_{i} / \beta_{i}}\right)^{m_{i}} \\
& \Phi_{\text {Ray }}(z)=\left(\frac{1 / \beta_{i}}{z+1 / \beta_{i}}\right) .
\end{aligned}
$$

Using these CFs, we are able to derive the pdfs of the total interference power $Y$ of multiple cochannel interferers for the two cochannel interference models. 


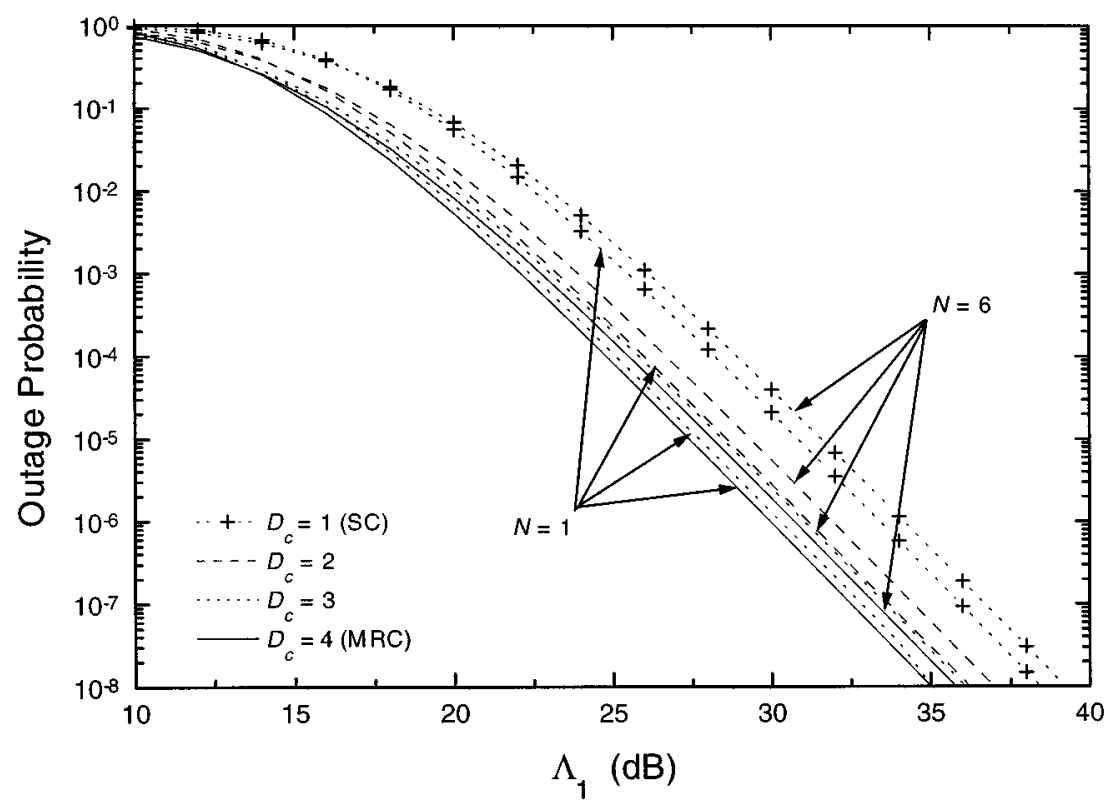

Fig. 2. Outage probability against average signal-to-average total interference power ratio $\Lambda_{1}$ for cochannel interference model 1 and different values of $D_{c}$ and $N$.

\section{A. Cochannel Interference Model 1}

In this cochannel interference model, we consider the case of $L$ cochannel interferers consisting of $L-N$ Nakagami- $m$ interferers and $N$ Rayleigh interferers. It is shown in the Appendix that, for integer Nakagami fading parameter $m_{i}$, the pdf of $Y$ for cochannel interference model 1 can be written as

$$
\begin{aligned}
p_{Y}(y)=\left(\prod_{j=1}^{L}\left(-a_{j}\right)^{m_{j}}\right) \\
\quad \times\left\{\sum_{k=1}^{L} \sum_{i=1}^{m_{k}} \frac{b_{k}^{i-1} y^{m_{k}-i}}{\left(m_{k}-i\right) !(i-1) !} \exp \left(a_{k} y\right)\right\}
\end{aligned}
$$

where $a_{h}=-m_{h} / \beta_{h}(h=1,2, \ldots, L)$ and

$$
b_{k}^{i-1}=\left.\frac{d^{i-1}}{d z^{i-1}}\left(\prod_{\substack{v \neq k \\ v=1}}^{L}\left(z-a_{v}\right)^{-m_{v}}\right)\right|_{z=a_{k}} .
$$

Note that $m_{h}=1$ for $h \geq L-N+1$ and all $a_{h}$ are assumed to be different.

\section{B. Cochannel Interference Model 2}

For cochannel interference model 2, we consider the case of $L$ independent cochannel interferers in which each interferer exhibits both Nakagami- $m$ pdf $p_{\mathrm{Nak}}\left(y_{i}\right)$ and Rayleigh pdf $p_{\text {Ray }}\left(y_{i}\right)$ alternatively. Here, we define a fading time-share factor $F, 0 \leq F \leq 1$. For a fraction of time $F$, the interferer is Nakagami- $m$ faded. For the remaining fraction of the time $1-F$, the interferer is Rayleigh faded. The net pdf of the power of the $i$ th interferer $Y_{i}$ is thus the weighted sum of the Nakagami- $m$ and Rayleigh pdfs as

$$
p_{Y_{i}}\left(y_{i}\right)=(F) p_{\mathrm{Nak}}\left(y_{i}\right)+(1-F) p_{\mathrm{Ray}}\left(y_{i}\right) \text {. }
$$

With the assumptions of 1) identical average power $\beta_{a}$ and fading parameter $m_{y}$ for all Nakagami- $m$ interferers, 2) identical average power $\beta_{b}$ for all Rayleigh interferers, and 3) identical fading time share factor $F$ for each of the $L$ cochannel interferers, the resulting $\mathrm{CF}$ of the sum of the powers of the $L$ cochannel interferers $Y$ can then be shown to be given by

$$
\begin{aligned}
\Phi_{Y}(z)= & \left\{F\left(\frac{m_{y} / \beta_{a}}{z+m_{y} / \beta_{a}}\right)^{m_{y}}+(1-F)\left(\frac{1 / \beta_{b}}{z+1 / \beta_{b}}\right)\right\}^{L} \\
= & \sum_{j=0}^{L}\left(\begin{array}{c}
L \\
j
\end{array}\right) F^{L-j}(1-F)^{j}\left(\frac{m_{y} / \beta_{a}}{z+m_{y} / \beta_{a}}\right)^{m_{y}(L-j)} \\
& \times\left(\frac{1 / \beta_{b}}{z+1 / \beta_{b}}\right)^{j} .
\end{aligned}
$$

After performing inverse Laplace transform on (11) and assuming integer values for $m_{y}$, the pdf of $Y$ for cochannel interference model 2 can be obtained as

$$
\begin{gathered}
p_{Y}(y)=\sum_{j=0}^{L}\left(\begin{array}{c}
L \\
j
\end{array}\right) F^{L-j}(1-F)^{j}\left(\frac{m_{y}}{\beta_{a}}\right)^{m_{y}(L-j)}\left(\frac{1}{\beta_{b}}\right)^{j} \\
\cdot\left(\sum_{k=1}^{2} \sum_{i=1}^{t_{k j}} \frac{e_{k j}^{i-1} y^{t_{k j}-i}}{\left(t_{k j}-i\right) !(i-1) !} \exp \left(c_{k} y\right)\right)
\end{gathered}
$$

where

$$
\begin{aligned}
c_{i} & = \begin{cases}-m_{y} / \beta_{a}, & i=1 \\
-1 / \beta_{b}, & i=2\end{cases} \\
t_{i j} & = \begin{cases}m_{y}(L-j), & i=1 \\
j, & i=2\end{cases} \\
e_{k j}^{i-1} & =\left.\frac{d^{i-1}}{d z^{i-1}}\left(\prod_{\substack{v \neq k \\
v=1}}^{2}\left(z-c_{v}\right)^{-t_{v j}}\right)\right|_{z=c_{k}} .
\end{aligned}
$$




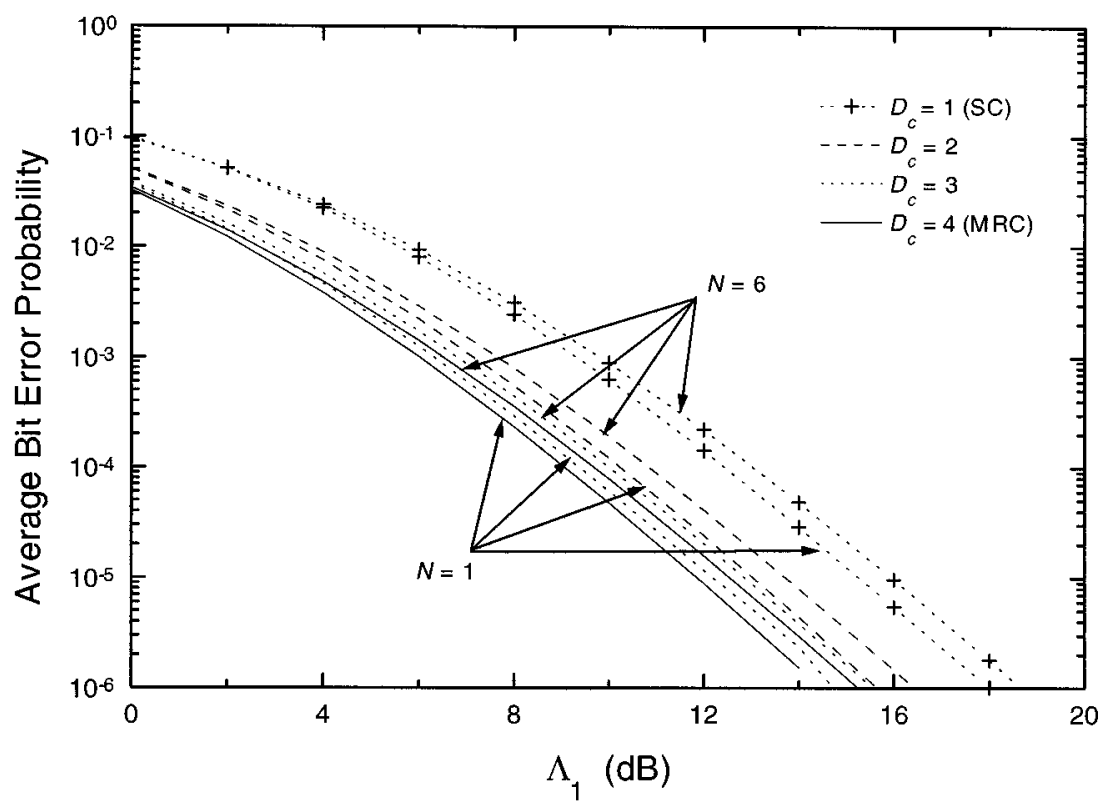

Fig. 3. Average BEP of DPSK against average signal-to-average total interference power ratio $\Lambda_{1}$ for cochannel interference model 1 and different values of $D_{c}$ and $N$.

\section{Derivations of the OUtage Probability AND AVERAGE BEP}

Assuming that the desired signal is modeled by Rayleigh distribution, the pdf of the instantaneous desired signal power $R$ is given by [2]

$$
p_{R}(r)=\frac{1}{\Omega} \exp \left(-\frac{r}{\Omega}\right)
$$

where $\Omega$ is the average desired signal power. The pdf of the instantaneous desired signal power $S$ of the combined signal at the output of the GSC can be deduced from [5] as

$$
\begin{aligned}
p_{S}(s)=\left(\begin{array}{c}
D \\
D_{c}
\end{array}\right)\{ & \frac{s^{D_{c}-1} e^{-s / \Omega}}{\Omega^{D_{c}}\left(D_{c}-1\right) !}+\frac{1}{\Omega} \sum_{l=1}^{D-D_{c}}(-1)^{D_{c}+l-1} \\
& \times\left(\begin{array}{c}
D-D_{c} \\
l
\end{array}\right) \cdot\left(\frac{D_{c}}{l}\right)^{D_{c}-1} \\
\times & {\left[\exp \left(-\left(\frac{1}{\Omega}+\frac{l}{D_{c} \Omega}\right) s\right)\right.} \\
& \left.\left.\quad-\sum_{n=0}^{D_{c}-2} \frac{1}{n !}\left(\frac{-l}{D_{c} \Omega}\right)^{n} s^{n} \exp \left(-\frac{s}{\Omega}\right)\right]\right\} .
\end{aligned}
$$

Note that the desired signals received on each branch are assumed to have the same $\Omega$.

\section{A. Outage Probability and Average BEP of GSC With Cochannel Interference Model 1}

Since $\gamma=S / Y$ is the instantaneous SIR at the output of the GSC, the pdf of $\gamma$ can be derived using

$$
p_{\gamma}(\gamma)=\int_{0}^{\infty} y p_{S}(y \gamma) p_{Y}(y) d y .
$$

Substituting (8) and (15) into (16) and using the following Laplace transform pair [12]:

$$
\int_{0}^{\infty} y^{A} \exp (-B y) d y=\frac{\Gamma(1+A)}{B^{1+A}}
$$

the pdf of $\gamma$ for the case of cochannel interference model 1 can be derived into closed form as

$$
\begin{aligned}
p_{\gamma}(\gamma)= & \left\{\prod_{j=1}^{L}\left(-a_{j}\right)^{m_{j}}\right\} \sum_{k=1}^{L} \sum_{i=1}^{m_{k}} \frac{b_{k}^{i-1}}{\left(m_{k}-i\right) !(i-1) !}\left(\begin{array}{c}
D \\
D_{c}
\end{array}\right) \\
& \cdot\left\{\frac{\Gamma\left(m_{k}-i+1+D_{c}\right)}{\Omega^{D_{c}}\left(D_{c}-1\right) !} \frac{\gamma^{D_{c}-1}}{\left(\gamma / \Omega-a_{k}\right)^{1+D_{c}+m_{k}-i}}\right. \\
& +\sum_{l=1}^{D-D_{c}} \frac{(-1)^{D_{c}+l-1}}{\Omega}\left(\begin{array}{c}
D-D_{c} \\
l
\end{array}\right)\left(\frac{D_{c}}{l}\right)^{D_{c}-1} \\
& \cdot\left[\frac{\Gamma\left(m_{k}-i+2\right)\left(\left(\frac{1}{\Omega}+\frac{l}{D_{c} \Omega}\right) \gamma-a_{k}\right)^{-m_{k}+i-2}}{}\right. \\
& -\sum_{n=0}^{D_{c}-2} \frac{1}{n !}\left(\frac{-l}{D_{c} \Omega}\right)^{n} \Gamma\left(n+m_{k}-i+2\right) \gamma^{n} \\
& \left.\left.\times\left(\frac{\gamma}{\Omega}-a_{k}\right)^{-\left(n+m_{k}-i+2\right)}\right]\right\}
\end{aligned}
$$

Having derived the pdf of $\gamma$ in (18), a closed-form expression for evaluating the outage probability and the average BEP is then derived as follows.

The outage probability is the probability of an interference power's exceeding the desired signal power divided by a power protection ratio $q$, and it can be evaluated using [11]

$$
P_{\mathrm{out}}=\int_{0}^{q} p_{\gamma}(\gamma) d \gamma
$$


Substituting (18) into (19) and using the following relation:

$$
\int_{0}^{A} \frac{y^{B-1}}{(C y+G)^{E}} d y=\frac{A^{B}}{B G^{E}}{ }^{2} F_{1}\left(\begin{array}{c}
B, E ; \\
B+1 ;
\end{array}-\frac{A C}{G}\right)
$$

the outage probability can then be simplified into a closed-form expression as shown in (21) at the bottom of the page, where ${ }_{2} F_{1}(\cdot)$ is the Gauss hypergeometric function [10].

For the derivation of the average BEP, the conditional BEP of a particular modulation scheme is required. In the application of DPSK signaling, the conditional BEP for a given SIR $\gamma$ is given by [13]

$$
P_{e}(\gamma)=\frac{1}{2} \exp (-\gamma)
$$

The average BEP $\bar{P}_{e}$ can then be evaluated by averaging the conditional BEP over pdf of $\gamma$ as

$$
\bar{P}_{e}=\int_{0}^{\infty} P_{e}(\gamma) p_{\gamma}(\gamma) d \gamma
$$

Substituting (18) and (22) into (23), the $\bar{P}_{e}$ can be derived into closed form as shown in (24) at the bottom of the page, where
$\Gamma(\cdot, \cdot)$ is the incomplete gamma function [10]. Note that the following derivation procedure has been used in the derivation of (24).

Let

$$
I=\int_{0}^{\infty} \frac{h^{A-1} e^{-G h}}{(B h+1)^{A+C}} d h
$$

Using variable transformation as $w=B h+1, I$ can be rewritten as

$$
I=\int_{1}^{\infty}\left(\frac{w-1}{B}\right)^{A-1} \frac{1}{B w^{A+C}} e^{-G(w-1) / B} d w .
$$

Using binomial expansion on the first term of the integrand in (26) and assuming integer values for $A, I$ can then be manipulated into closed form as

$$
\begin{aligned}
I= & \left(\frac{1}{B}\right) \sum_{r=0}^{A}\left(\begin{array}{c}
A-1 \\
r
\end{array}\right)(-1)^{r} e^{G / B} \\
& \times \int_{1}^{\infty} w^{-(1+r+C)} e^{-G w / B} d w
\end{aligned}
$$

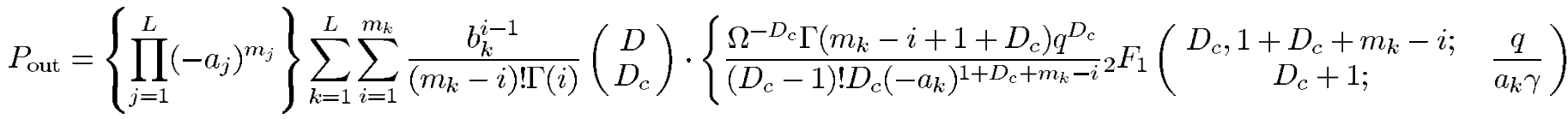

$$
\begin{aligned}
& +\sum_{l=1}^{D-D_{c}} \frac{(-1)^{D_{c}+l-1}}{\Omega}\left(\begin{array}{c}
D-D_{c} \\
l
\end{array}\right)\left(\frac{D_{c}}{l}\right)^{D_{c}-1} \\
& \cdot\left[\frac{q \Gamma\left(m_{k}-i+2\right)}{\left(-a_{k}\right)^{m k-i+2}}{ }_{2} F_{1}\left(\begin{array}{c}
1, m_{k}-i+2 ; \\
2 ;
\end{array} ;\left(\frac{1}{\Omega}+\frac{l}{D_{c} \Omega}\right) \frac{q}{a_{k}}\right)\right. \\
& -\sum_{n=0}^{D_{c}-2} \frac{1}{n !}\left(\frac{-l}{D_{c} \Omega}\right)^{n} \frac{q^{n+1} \Gamma\left(n+m_{k}-i+2\right)}{(n+1)\left(-a_{k}\right)^{n+m_{k}-i+2}} \\
& \left.\left.{ }_{2} F_{1}\left(\begin{array}{cc}
n+1, n+m_{k}-i+2 ; & \frac{q}{\Omega a_{k}}
\end{array}\right)\right]\right\}
\end{aligned}
$$

$$
\begin{aligned}
\bar{P}_{e}= & \frac{1}{2}\left\{\prod_{j=1}^{L}\left(-a_{j}\right)^{m_{j}}\right\} \sum_{k=1}^{L} \sum_{i=1}^{m_{k}} \frac{b_{k}^{i-1}}{\left(m_{k}-i\right) ! \Gamma(i)}\left(\begin{array}{c}
D \\
D_{c}
\end{array}\right) \\
& \cdot\left\{\sum_{r=0}^{D_{c}-1}\left(\begin{array}{c}
D_{c}-1 \\
r
\end{array}\right) \frac{\Gamma\left(m_{k}-i+1+D_{c}\right) \Gamma\left(-r-1-m_{k}+i,-\Omega a_{k}\right)}{\left(D_{c}-1\right) !\left(-a_{k}\right)^{-r} \exp \left(\Omega a_{k}\right)(\Omega)^{-\left(r+1+m_{k}-i\right)}}\right. \\
+ & \sum_{l=1}^{D_{-} D_{c}} \frac{(-1)^{D_{c}+l-1}}{\Omega}\left(\begin{array}{c}
D-D_{c} \\
l
\end{array}\right)\left(\frac{D_{c}}{l}\right)^{D_{c}-1} \\
& \cdot\left[\Gamma\left(m_{k}-i+2\right)\left(\frac{1}{\Omega}+\frac{l}{D_{c} \Omega}\right)^{i-m_{k}-2}\right. \\
& \cdot \exp \left(-a_{k} /\left(\frac{1}{\Omega}+\frac{l}{D_{c} \Omega}\right)\right) \Gamma\left(i-1-m_{k}, \frac{-a_{k}}{\left(1 / \Omega+l / D_{c} \Omega\right)}\right)-\sum_{n=0}^{D_{c}-2} \frac{\Omega}{n !}\left(\frac{-l}{D_{c}}\right)^{n} \\
& \left.\cdot \sum_{t=0}^{n}\left(\begin{array}{c}
n \\
t
\end{array}\right) \frac{\Gamma\left(n+m_{k}-i+2\right) \Gamma\left(-t+i-1-m_{k},-a_{k} \Omega\right)}{e^{\Omega a_{k}} a_{k}^{-t} \Omega^{-\left(t+m_{k}-i+1\right)}}\right]
\end{aligned}
$$




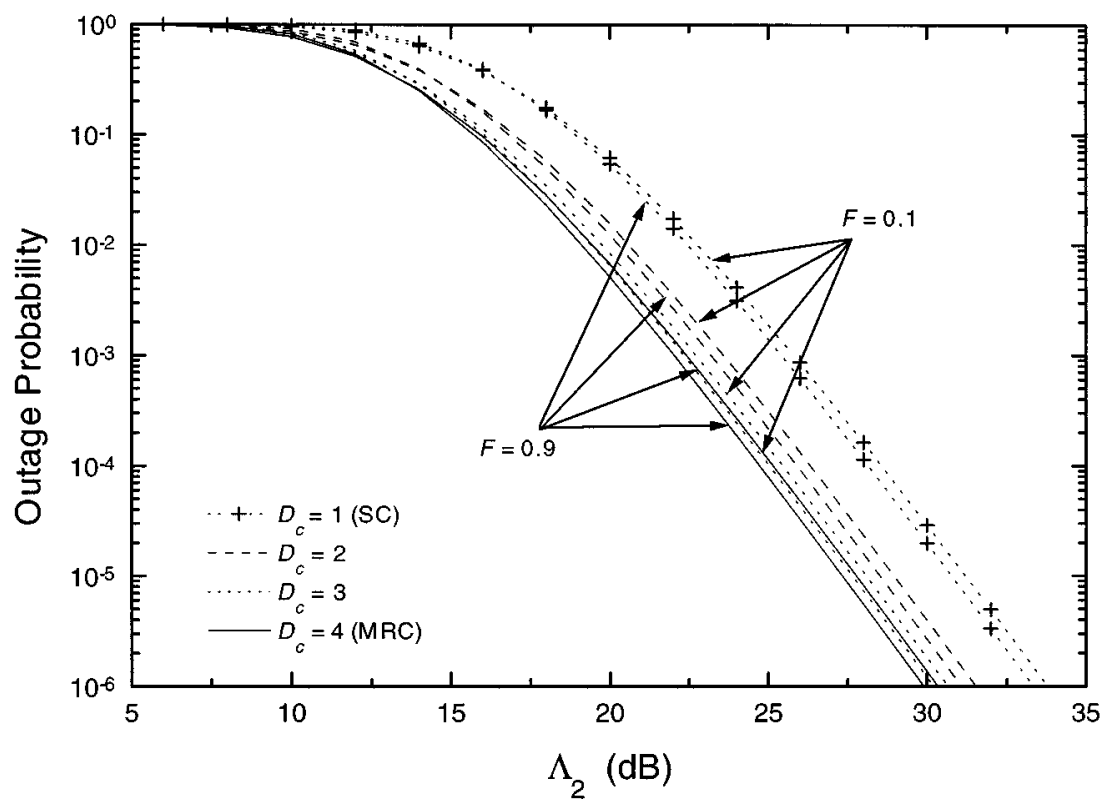

Fig. 4. Outage probability against average signal-to-average total interference power ratio $\Lambda_{2}$ for cochannel interference model 2 and different values of $D_{c}$ and F.

$$
\begin{aligned}
= & \left(\frac{1}{B}\right) \sum_{r=0}^{A-1}\left(\begin{array}{c}
A-1 \\
r
\end{array}\right)(-1)^{r} e^{G / B}\left(\frac{G}{B}\right)^{r+C} \\
& \times \Gamma\left(-r-C, \frac{G}{B}\right) .
\end{aligned}
$$

\section{B. Outage Probability and Average BEP of GSC With Cochannel Interference Model 2}

For the interference model 2 , the derivation procedure for the case of cochannel interference model 1 can also be applied. Substituting (12) and (15) into (16) and using the Laplace transform pair in (17), the pdf of $\gamma$ for the case of cochannel interference model 2 after some manipulations lead to

$$
\begin{aligned}
& p_{\gamma}(\gamma)=\sum_{j=0}^{L}\left(\begin{array}{c}
L \\
j
\end{array}\right) F^{L-j}(1-F)^{j}\left(\frac{m_{y}}{\beta_{a}}\right)^{m_{y}(L-j)}\left(\frac{1}{\beta_{b}}\right)^{j} \\
& \cdot \sum_{k=1}^{2} \sum_{i=1}^{t_{k j}} \frac{e_{k j}^{i-1}}{(i-1) !\left(t_{k j}-i\right) !}\left(\begin{array}{c}
D \\
D_{c}
\end{array}\right) \\
& \times\left\{\frac{\Gamma\left(t_{k j}-i+1+D_{c}\right)}{\Omega^{D_{c}}\left(D_{c}-1\right) !} \cdot \frac{\gamma^{D_{c}-1}}{\left(\gamma / \Omega-c_{k}\right)^{1+D_{c}+t_{k, j}-i}}\right. \\
& +\sum_{l=1}^{D-D_{c}} \frac{(-1)^{D_{c}+l-1}}{\Omega}\left(\begin{array}{c}
D-D_{c} \\
l
\end{array}\right)\left(\frac{D_{c}}{l}\right)^{D_{c}-1} \\
& \cdot\left[\Gamma\left(t_{k j}-i+2\right)\left(\left(\frac{1}{\Omega}+\frac{l}{D_{c} \Omega}\right) \gamma-c_{k}\right)^{-t_{k j}+i-2}\right. \\
& -\sum_{n=0}^{D_{c}-2} \frac{1}{n !}\left(\frac{-l}{D_{c} \Omega}\right)^{n} \Gamma\left(n+t_{k j}-i+2\right) \gamma^{n} \\
& \left.\left.\times\left(\frac{\gamma}{\Omega}-c_{k}\right)^{-\left(n+t_{k ;}-i+2\right)}\right]\right\} \text {. }
\end{aligned}
$$

Using the relation in (20) and substituting (28) into (19), the outage probability for the case of cochannel interference model 2 can be obtained. In addition, by substituting (22) and (28) into (23), and after further manipulations, the average BEP for the case of cochannel interference model 2 can also be derived straightforwardly. Details of the derivations are omitted for the sake of brevity.

\section{NumERICAL RESUlts}

In this section, numerical results are presented on the outage and the average bit error probabilities of GSC diversity systems for the two cochannel interference models. The power protection ratio, the number of available diversity signals, and the number of cochannel interferers are equal to $q=18 \mathrm{~dB}, D=4$, and $L=6$, respectively.

Fig. 2 shows the outage probability versus the average desired signal to average total interference power ratio $\Lambda_{1}=\Omega /\left(\beta_{1}+\cdots+\beta_{L}\right)$ for cochannel interference model 1 and different values of $D_{c}$ and $N$. Fig. 3 provides the average BEP of DPSK as a function of $\Lambda_{1}$ for cochannel interference model 1 and different values of $D_{c}$ and $N$. Note that $m_{i}=3(i \leq L-N), m_{i}=1(i>L-N)$ and $\left\{\beta_{i}\right\}=\{12.1,9.3,6.1,5.7,1.7,1.3\}$ are assumed in Figs. 2 and 3. One can see that a desired outage probability or average BEP can be achieved at smaller $\Lambda_{1}$ for decreasing $N$ or increasing $D_{c}$. Note also that GSC becomes MRC and SC when $D_{c}=D$ and 1 , respectively.

In Fig. 4, the outage probability is depicted in relation to the average desired signal to average total interference power ratio $\Lambda_{2}$ defined as $\Lambda_{2}=\Omega / L\left(F \beta_{a}+(1-F) \beta_{b}\right)$ for interference model 2 and different values of $D_{c}$ and $F$. In Fig. 5, the average BEP of DPSK is plotted against $\Lambda_{2}$ for different values of $D_{c}$ and $F$. We assume in Figs. 4 and 5 that $m_{y}=3, \beta_{a}=4$, and $\beta_{b}=4$. It can be seen that the outage probability or the average 


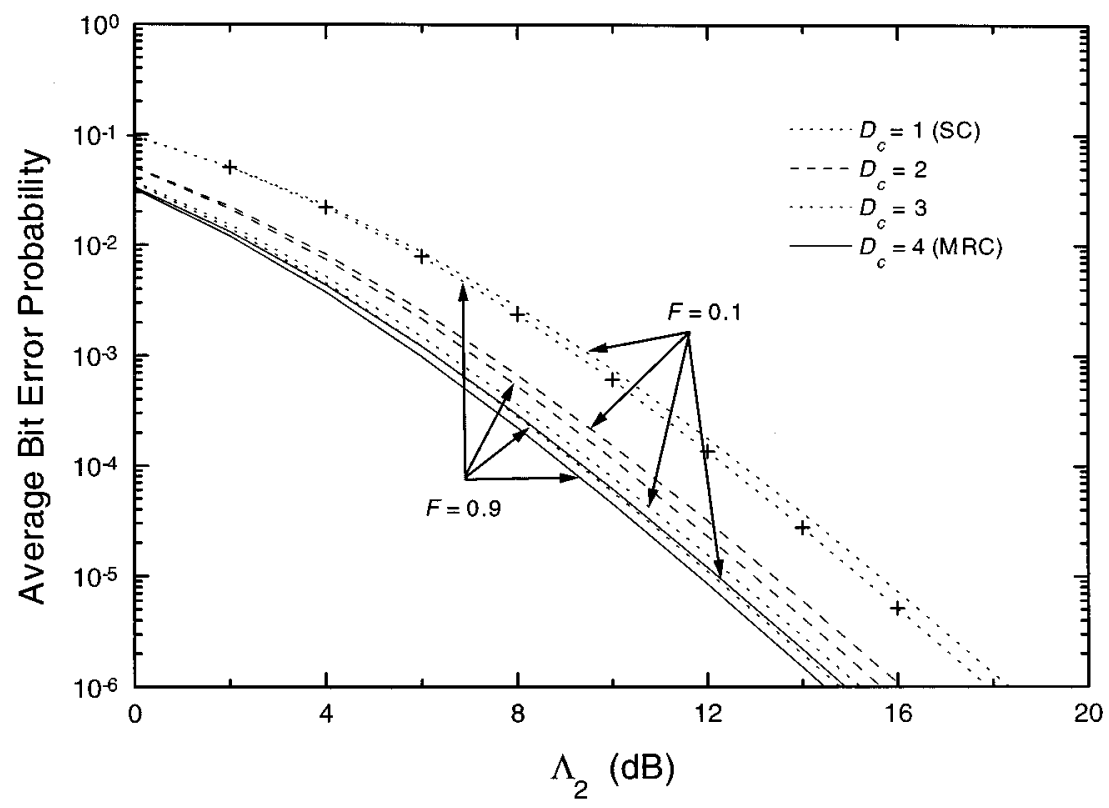

Fig. 5. Average BEP of DPSK against average signal-to-average total interference power ratio $\Lambda_{2}$ for cochannel interference model 2 and different values of $D_{c}$ and $F$.

BEP decreases with increasing $D_{c}$ and $F$. From all the above figures, we know that in the presence of multiple cochannel interference, GSC can also achieve much better performance than $\mathrm{SC}$, and similar performance as MRC.

\section{CONCLUSION}

In this paper, we studied GSC for mobile radio systems in the presence of Rayleigh desired signal and two cochannel interference models. The desired signals and the cochannel interferers received on each branch are assumed to be independent. The pdfs of the instantaneous SIR at the output of GSC have been derived for the two cochannel interference models. Using these new SIR pdfs, closed-form expressions for the outage probability and the average BEP, which provide a convenient tool for performance analysis, were then derived. The effects of various system parameters on the outage probability and the average $\mathrm{BEP}$ were also presented.

\section{APPENDIX}

\section{DERIVATION OF THE DENSITY FUNCTION OF $Y$ FOR COCHANNEL INTERFERENCE MODEL 1}

In this Appendix, we show that the pdf of the resultant cochannel interfering power $Y$ is given by (8) for cochannel interference model 1 . Assuming the presence of $L$ independent cochannel interferers' being either Nakagami- $m$ and Rayleigh faded, the power of the resultant interfering signals $Y$ can be written as

$$
Y=Y_{\text {Nak }}+Y_{\text {Ray }}
$$

where $Y_{\text {Nak }}=Y_{1}+\cdots+Y_{L-N}$ and $Y_{\text {Ray }}=Y_{L-N+1}+\cdots+Y_{L}$ are the sums of the powers of the $L-N$ Nakagami- $m$ interferers and the $N$ Rayleigh interferers, respectively. Note again that $Y_{i}(i=1,2, \ldots, L)$ is the power of the $i$ th interferer. From (A1), it can be deduced that the $\mathrm{CF}$ of $Y, \Phi_{Y}(z)$ can be given in terms of $\Phi_{\mathrm{Nak}}(z)$ and $\Phi_{\mathrm{Ray}}(z)$ as

$$
\begin{aligned}
\Phi_{Y}(z)= & \Phi_{\mathrm{Nak}}(z) \times \Phi_{\mathrm{Ray}}(z) \\
= & \left(\prod_{i=1}^{L-N}\left(\frac{m_{i} / \beta_{i}}{z+m_{i} / \beta_{i}}\right)^{m_{i}}\right) \\
& \times\left(\prod_{i=L-N+1}^{L}\left(\frac{1 / \beta_{i}}{z+1 / \beta_{i}}\right)\right)=\prod_{i=1}^{L}\left(\frac{-a_{i}}{z-a_{i}}\right)^{m_{i}}
\end{aligned}
$$

where $a_{i}=-m_{i} / \beta_{i}(i=1,2, \ldots, L)$. Note that $m_{i}=1$ for $i \geq L-N+1$ (i.e., Rayleigh fading). In addition, all $a_{i}$ are assumed to be different. Taking the inverse Laplace transform of (A2) as in ([11, Appendix]), one obtains (8).

\section{ACKNOWLEDGMENT}

The authors would like to thank the anonymous reviewers for their valuable comments and suggestions, which enhanced the quality of this paper.

\section{REFERENCES}

[1] W. C. Jakes Jr., Microwave Mobile Communication. New York: Wiley, 1974.

[2] J. Proakis, Digital Communications. New York: McGraw-Hill, 1995.

[3] N. Kong, T. Eng, and L. B. Milstein, "Selection combining scheme for Rake receiver," in Proc. Int. Conf. Univ. Personal Comm., Nov. 1995, pp. 426-429.

[4] T. Eng, N. Kong, and L. B. Milstein, "Comparison of diversity combining techniques for Rayleigh-fading channels," IEEE Trans. Commun., vol. 44, pp. 1117-1128, Sept. 1996.

[5] M.-S. Alouini and M. K. Simon, "An MGF-based performance analysis of generalized selection combining over Rayleigh fading channels," IEEE Trans. Commun., vol. 48, pp. 401-415, Mar. 2000.

[6] - "Performance of coherent receivers with hybrid SC/MRC over Nakagami- $m$ fading channels," IEEE Trans. Veh. Technol., vol. 48, pp. 1155-1164, July 1999. 
[7] _ - "Application of the Dirichlet transformation to the performance evaluation of generalized selection combining over Nakagami- $m$ fading channels," J. Commun. Networks, vol. 1, no. 1, pp. 5-13, Mar. 1999.

[8] Y. Ma and C. C. Chai, "Unified error probability analysis for generalized selection combining in Nakagami fading channels," IEEE J. Select. Areas Commun., vol. 18, pp. 2198-2210, Nov. 2000.

[9] M. Nakagami, "The m-distribution: A general formula of intensity distribution of rapid fading," in Statistical Methods in Radio Wave Propagation, W. G. Hoffman, Ed. New York: Pergamon, 1960.

[10] M. Abramowitz and I. A. Stegun, Handbook of Mathematical Functions with Formulas, Graphs, and Mathematical Tables, 9th ed. New York: Dover, 1970.

[11] A. A. Abu-Dayya and N. C. Beaulieu, "Outage probabilities of cellular mobile radio systems with multiple Nakagami interferers," IEEE Trans. Veh. Technol., vol. 40, pp. 757-768, Nov. 1991

[12] A. P. Prudnikov, Y. A. Brychkov, and O. I. Marichev, Integrals and Series: Direct Laplace Transforms. London, U.K.: Gordon and Breach, 1992.

[13] V. A. Aalo and J. Zhang, "On the effect of cochannel interference on average error rates in Nakagami-fading channels," IEEE Trans. Commun. Lett., vol. 3, pp. 136-138, May 1999.

[14] J. H. Winters, "Optimum combining in digital mobile radio with cochannel interference," IEEE J. Select. Areas Commun., vol. 2, pp. 528-539, July 1984.

[15] V. A. Aalo and J. Zhang, "Performance analysis of maximal ratio combining in the presence of multiple equal-power cochannel interferers in a Nakagami fading channel," IEEE Trans. Veh. Technol., vol. 50, pp. 497-503, Mar. 2001.

[16] J. Reig, N. Cardona, and L. Rubio, "Performance of radio cellular systems using maximal ratio combining in a correlated Nakagami fading with multiple interferers," in Proc. VTC'99, May 1999, pp. 2358-2362

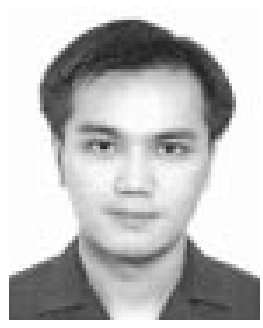

Chi Ming Lo received the B.Eng. (Hons.) degree in information systems engineering from the Imperial College of Science, Technology and Medicine, London, U.K., in 1993 and the M.A.Sc. degree in electrical engineering from the University of British Columbia, Vancouver, BC, Canada, in 1996. He is currently pursuing the Ph.D. degree in the Department of Electrical and Electronic Engineering, The University of Hong Kong, Hong Kong, China

His main research interests include the performance analysis of wireless digital communication

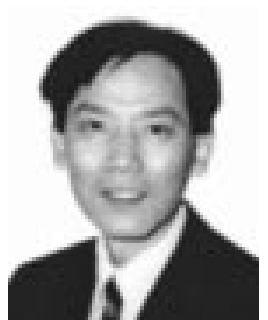

Wong Hing Lam (S'86-M'87-SM'96) was born in Hong Kong on March 7, 1960. He received the B.Sc. degree in computer and communication engineering from the University of Essex, U.K. in 1983, the M.Sc. degree in telecommunication engineering from the Imperial College, University of London, U.K., in 1984, and the Ph.D. degree from the University of Southampton, U.K.

He was sponsored by and collaborated with British Telecom Research Laboratory (BTRL), Plessey Research Laboratory (U.K.), and Bell Labs to work on the pan-European digital cellular mobile radio communications systems, which is known as GSM. He was a member of the Mobile Radio Research Group and specialized in digital cellular mobile radio communications including GSM, CDMA, and RACE. In 1988, he joined STL (STC Laboratory), Harlow, U.K., where he was responsible for the UK DTI-LINK project "phones on the move" of DTI, microcellular project, formulation of the personal communication systems $(\mathrm{PCN})$ architecture, and subsequently winning the operation license application of the British PCN. In 1989, he was with the Pan-European Digital Cellular Infrastructure R\&D center, Motorola Limited, Swindon, U.K., where he was responsible for the development and validation of the GSM systems, radio resource network management project, which encompassed radio propagation measurements and prediction, geographical information systems (GIS), GSM and PCN radio frequency planning, and radio network planning. In 1991, he joined the Department of Electrical and Electronic Engineering, University of Hong Kong, where he is currently heading a group of researchers in the field of mobile radio communications and intelligent transport systems (ITS). He has been very active locally and internationally. He has published more than 50 technical publications in the field of digital cellular mobile radio communications and ITS. He was the Chairman and Organizer of a series of regional conferences on mobile radio communications and a Distinguished Speaker and Chairperson at several international conferences and meetings. His current research interest include UMTS, FLMTS, CDMA, radio propagation, radio resource optimization, wireless LAN and WAN, broadband ISDN, Global Positioning System, and ITS.

Dr. Lam received the SERC CASE award from the University of Southampton. 